\title{
THE H-FUNCTION TRANSFORM II
}

\author{
K. C. GUPTA and (Mrs) P. K. MITTAL
}

(Received 6 August 1969)

Communicated by E. Strzelecki

\begin{abstract}
In this paper first we prove the uniqueness theorem for an integral transform whose kernel is $\boldsymbol{H}$-function. Later on we establish a new and interesting theorem concerning this transform and a generalized Laplace transform whose kernel is Meijer's $G$-function.
\end{abstract}

\section{Introduction}

This paper is sequel to a recent paper [3] in this journal. There we had defined the $H$-function transform by the integral equation:

$\phi(s)=H\left\{f(t) ;\left(a_{1}, \alpha_{1}\right), \cdots,\left(a_{p}, \alpha_{p}\right) ;\left(b_{1}, \beta_{1}\right), \cdots,\left(b_{q}, \beta_{q}\right) ; s\right\}$

$$
=s \int_{0}^{\infty} H_{p, q}^{m, n}\left[s t \mid \begin{array}{l}
\left(a_{1}, \alpha_{1}\right), \cdots,\left(a_{p}, \alpha_{p}\right) \\
\left(b_{1}, \beta_{1}\right), \cdots,\left(b_{q}, \beta_{q}\right)
\end{array}\right] f(t) d t,
$$

where the $H$-function is defined and represented as follows:

$$
\begin{aligned}
H_{p, q}^{m, n}\left[x \mid \begin{array}{l}
\left(a_{1}, \alpha_{1}\right), \cdots,\left(a_{p}, \alpha_{p}\right) \\
\left(b_{1}, \beta_{1}\right), \cdots,\left(b_{q}, \beta_{q}\right)
\end{array}\right] \\
\qquad=\left(\frac{1}{2 \pi i}\right) \int_{L} \frac{\prod_{j=1}^{m} \Gamma\left(b_{j}-\beta_{j} \xi\right) \prod_{j=1}^{n} \Gamma\left(1-a_{j}+\alpha_{j} \xi\right)}{\prod_{j=m+1}^{q} \Gamma\left(1-b_{j}+\beta_{j} \xi\right) \prod_{j=n+1}^{p} \Gamma\left(a_{j}-\alpha_{j} \xi\right)} x^{\xi} d \xi .
\end{aligned}
$$

The conditions satisfied by the parameters of the $H$-function, its asymptotic expansion and some of its important properties have already been given in the paper referred to above.

The following notations will be used throughout this paper:

1) $\left(a_{i}+b, \alpha_{i}\right)_{m, n}(n>m)$ will stand for $n-m+1$ pairs $\left(a_{m}+b, \alpha_{m}\right)$, $\left(a_{m+1}+b, \alpha_{m+1}\right), \cdots,\left(a_{n}+b, \alpha_{n}\right)$. Thus $\left(a_{i}, \alpha_{i}\right)_{1, n}$ will stand for $n$ pairs $\left(a_{1}, \alpha_{1}\right)$, $\cdots,\left(a_{n}, \alpha_{n}\right)$ and so on.

2) $f(x) \in A(\alpha, \beta, y)$ would mean that

$$
\left.\begin{array}{rl}
f(x) & =O\left(x^{\alpha}\right) \text { for small } x, \\
& =O\left(x^{\beta} e^{-y x}\right) \text { for large } x .
\end{array}\right\}
$$


The results given below will be required later on $[5$, p. $401 ; 4$, p. $50 ; 6$, p. 374]

$$
\begin{aligned}
& G_{\gamma, \boldsymbol{\delta}}^{\alpha, \boldsymbol{\beta}}\left[x \mid \begin{array}{l}
a_{1}, \cdots, a_{\gamma} \\
b_{1}, \cdots, b_{\delta}
\end{array}\right] \\
& =(2 \pi)^{(1-S)(\alpha+\beta-\gamma / 2-\delta / 2)} S_{1}^{\left(\sum b_{i}-\sum_{1}^{\gamma} a_{i}+\gamma / 2-\delta / 2+1\right)} \\
& \times G_{S \gamma, S \delta}^{S \alpha, S \beta}\left[\begin{array}{l|l}
x^{S} S^{S(\gamma-\delta)} & \begin{array}{l}
\Delta\left(a_{1}, S\right), \cdots, \Delta\left(a_{\gamma}, S\right) \\
\Delta\left(b_{1}, S\right), \cdots, \Delta\left(b_{\delta}, S\right)
\end{array}
\end{array}\right]
\end{aligned}
$$

where $\Delta(a, S)$ stands for quantities $a / S,(a+1) / S, \cdots,(a+S-1) / S$.

$$
H_{p, q}^{m, n}\left[x \mid \begin{array}{c}
\left(a_{1}, 1\right), \cdots,\left(a_{p}, 1\right) \\
\left(b_{1}, 1\right), \cdots,\left(b_{q}, 1\right)
\end{array}\right]=G_{p, q}^{m, n}\left[x \mid \begin{array}{l}
a_{1}, \cdots, a_{p} \\
b_{1}, \cdots, b_{q}
\end{array}\right]
$$

where the function on the right denotes Meijer's $G$-function [1, p. 207]

$$
H_{p, q}^{m, n}\left[x \mid \begin{array}{c}
\left(a_{1}, \alpha_{1}\right), \cdots,\left(a_{p}, \alpha_{p}\right) \\
\left(b_{1}, \beta_{1}\right), \cdots,\left(b_{q}, \beta_{q}\right)
\end{array}\right]=c H_{p, q}^{m, n}\left[x^{c} \mid \begin{array}{c}
\left(a_{1}, c \alpha_{1}\right), \cdots,\left(a_{p}, c \alpha_{p}\right) \\
\left(b_{1}, c \beta_{1}\right), \cdots,\left(b_{q}, c \beta_{q}\right)
\end{array}\right]
$$

where $c>0$.

If one of $\left(a_{i}, \alpha_{i}\right)(i=1, \cdots, n)$, is equal to one of $\left(b_{j}, \beta_{j}\right)(j=m+1, \cdots, q)$ [or one of the $\left(b_{i}, \beta_{i}\right)(i=1, \cdots, m)$ is equal to one of the $\left(a_{j}, \alpha_{j}\right)(j=$ $n+1, \cdots, p)]$, then the $H$-function reduces to one of the lower order, that is $p, q$ and $n$ (or $m$ ) decreases by unity; we give below one such reduction formula:

$$
\begin{aligned}
H_{p, q}^{m, n}\left[x \mid \begin{array}{l}
\left(a_{1}, \alpha_{1}\right),\left(a_{2}, \alpha_{2}\right), \cdots,\left(a_{p}, \alpha_{p}\right) \\
\left(b_{1}, \beta_{1}\right), \cdots,\left(b_{q-1}, \beta_{q-1}\right),\left(a_{1}, \alpha_{1}\right)
\end{array}\right] \\
=H_{n-1, q-1}^{m, n-1}\left[\begin{array}{l}
\left.x \mid \begin{array}{l}
\left(a_{2}, \alpha_{2}\right), \cdots,\left(a_{p}, \alpha_{p}\right) \\
\left(b_{1}, \beta_{1}\right), \cdots,\left(b_{q-1}, \beta_{q-1}\right)
\end{array}\right]
\end{array}\right.
\end{aligned}
$$

Other reduction formulae are similar.

$$
\begin{aligned}
& \int_{0}^{\infty} x^{\eta-1} H_{p, q}^{m, n}\left[z x^{\sigma} \mid \begin{array}{c}
\left(a_{i}, \alpha_{i}\right)_{1, p} \\
\left(b_{i}, \beta_{i}\right)_{1, q}
\end{array}\right] H_{r, l}^{k, f}\left[s x \mid \begin{array}{l}
\left(c_{i}, \gamma_{i}\right)_{1, r} \\
\left(d_{i}, \delta_{i}\right)_{1, l}
\end{array}\right] d x \\
& =s^{-\eta} H_{p+l, q+r}^{m+f, n+k}\left[z s^{-\sigma} \mid \begin{array}{l}
\left(a_{i}, \alpha_{i}\right)_{1, n},\left(1-d_{i}-\eta \delta_{i}, \sigma \delta_{i}\right)_{1, l},\left(a_{i}, \alpha_{i}\right)_{n+1, p} \\
\left(b_{i}, \beta_{i}\right)_{1, m},\left(1-c_{i}-\eta \gamma_{i}, \sigma \gamma_{i}\right)_{1, r},\left(b_{i}, \beta_{i}\right)_{m+1, q}
\end{array}\right]
\end{aligned}
$$

where

$$
\begin{array}{cc}
R\left(\eta+\sigma b_{h} / \beta_{h}+d_{i} / \delta_{i}\right)>0 & (h=1, \cdots, m ; i=1, \cdots, k), \\
R\left(\eta+\left(c_{j}-1\right) / \gamma_{j}+\sigma\left(a_{i}-1\right) / \alpha_{i}\right)<0 & (j=1, \cdots, f ; i=1, \cdots, n), \\
A=\sum_{1}^{n}\left(\alpha_{j}\right)-\sum_{n+1}^{p}\left(\alpha_{j}\right)+\sum_{1}^{m}\left(\beta_{j}\right)-\sum_{m+1}^{q}\left(\beta_{j}\right)>0, \\
B=\sum_{1}^{f}\left(\gamma_{i}\right)-\sum_{j+1}^{r}\left(\gamma_{i}\right)+\sum_{1}^{k}\left(\delta_{j}\right)-\sum_{k+1}^{l}\left(\delta_{j}\right)>0, \\
|\arg z|<\frac{1}{2} A \pi,|\arg s|<\frac{1}{2} B \pi .
\end{array}
$$


To prove the uniqueness of theorem for the $H$-function transform we prove the following lemma:

LEMMA. If

$$
\int_{0}^{\infty} t^{\rho} H_{p, q}^{m, n}\left[s t \mid \begin{array}{l}
\left(a_{i}, \alpha_{i}\right)_{1, p} \\
\left(b_{i}, \beta_{i}\right)_{1, q}
\end{array}\right] f(t) d t=0
$$

then

$$
f(t) \equiv 0,
$$

provided that $f(t)$ is continuous in $t>0, f(t) \in A(\alpha, \beta, y), R(y)>0$,

and

$$
\begin{array}{ll}
\lambda=\sum_{1}^{n}\left(\alpha_{j}\right)-\sum_{n+1}^{p}\left(\alpha_{j}\right)+\sum_{1}^{m}\left(\beta_{j}\right)-\sum_{m+1}^{q}\left(\beta_{j}\right)>0, & |\arg s|<\frac{1}{2} \lambda \pi, \\
R\left(\rho+\alpha+b_{j} / \beta_{j}+1\right)>0 & (j=1, \cdots, m), \\
R\left(b_{i} / \beta_{i}+\left(1-b_{j}\right) / \beta_{j}\right)>0 & (j=m+1, \cdots, q ; i=1, \cdots, m)
\end{array}
$$

$$
R\left(\left(a_{i}-1\right) / \alpha_{i}-a_{j} / \alpha_{j}\right)<0 \quad(i=1, \cdots, n ; j=n+1, \cdots, p) .
$$

Proof. Multiplying (2.1) by

$$
\begin{aligned}
& \text { (A) } s^{n-1} H_{p, c+q}^{c+q-m, p-n} \\
& \times\left[s\left(\frac{z}{c}\right)^{N c} \mid \begin{array}{l}
\left(1-a_{i}-\eta \alpha_{i}, \alpha_{i}\right)_{n+1, p},\left(1-a_{i}-\eta \alpha_{i}, \alpha_{i}\right)_{1, n} \\
(0, N),\left(\frac{1}{c}, N\right), \cdots,\left(\frac{c-1}{c}, N\right),\left(1-b_{i}-\eta \beta_{i}, \beta_{i}\right)_{m+1, q}, \\
\left(1-b_{i}-\eta \beta_{i}, \beta_{i}\right)_{1, m}
\end{array}\right]
\end{aligned}
$$

where $c N>\lambda,|\arg z|<\frac{1}{2} \pi(1-(\lambda / N c)), R\left(\eta+\left(b_{j} / \beta_{j}\right)\right)>0(j=1, \cdots, m)$, and integrating it with respect to $s$ between the limits 0 to $\infty$ we get

$$
\begin{aligned}
& \int_{0}^{\infty} s^{\eta-1} H_{p, c+q}^{c+q-m, p-n}\left[s\left(\frac{z}{c}\right)^{N c} \mid \cdots \cdots \cdots\right] \\
& \times\left\{\int_{0}^{\infty} t^{\rho} H_{p, q}^{m, n}\left[s t \mid \begin{array}{c}
\left(a_{i}, \alpha_{i}\right)_{1, p} \\
\left(b_{i}, \beta_{i}\right)_{1, q}
\end{array}\right] f(t) d t\right\} d s=0
\end{aligned}
$$

where the parameters of one of the $H$-function shown by ..... are same as given in (A).

On interchanging the order of integration in (2.3), which is justified on account of absolute convergence of the integrals under the conditions imposed, and evaluating the $s$-integral therein with the help of (1.7) we get by virtue of (1.6)

$$
\int_{0}^{\infty} t^{\rho} f(t) H_{0, c}^{c, 0}\left[\left(\frac{z}{c}\right)^{N c} \frac{1}{t} \mid(0, N),\left(\frac{1}{c}, N\right), \cdots,\left(\frac{c-1}{c}, N\right)\right] d t=0
$$


Now the $H$-function in (2.4) reduces to Meijer's $G$-function [1, p. 207] with the help of (1.4) and (1.5) its value being equal to

$$
\frac{1}{N} G_{0, c}^{c, 0}\left[t^{-1 / N}\left(\frac{z}{c}\right)^{c} \mid 0, \frac{1}{c}, \cdots, \frac{c-1}{c}\right] .
$$

Also the expression given by (B) reduces to the following expression with the help of (1.3):

$$
\frac{1}{N} c^{-\frac{1}{2}}(2 \pi)^{\frac{1}{2}(c-1)} G_{0,1}^{1,0}\left[z t^{-1 / N c} \mid 0\right],
$$

but the $G$-function involved in (C) is equal to

Therefore (2.4) reduces to

$$
e^{-z t^{-1 / N c}} \text {. }
$$

$$
\int_{0}^{\infty} t^{\rho} f(t) \exp \left(-z t^{-1 / N c}\right) d t=0
$$

or

$$
\int_{0}^{\infty} t^{-N_{c}-1-\rho / N c} f\left(t^{-N c}\right) \exp (-z t) d t=0
$$

On applying Lerch's theorem [2, p. 339] to (2.5), we get

$$
f\left(t^{-N c}\right) \equiv 0 \text {. }
$$

From (2.6) it follows that $f(t) \equiv 0$. The uniqueness theorem for the $H$-function transform follows as direct consequence of the above lemma.

The uniqueness theorem

If $f_{1}(t)$ and $f_{2}(t)$ are continuous in $t \geqq 0$ and

$$
\int_{0}^{\infty} H_{p, q}^{m, n}\left[s t \mid \begin{array}{l}
\left(a_{i}, \alpha_{i}\right)_{1, p} \\
\left(b_{i}, \beta_{i}\right)_{1, q}
\end{array}\right] f_{1}(t) d t=\int_{0}^{\infty} H_{p, q}^{m, n}\left[s t \mid \begin{array}{c}
\left(a_{i}, \alpha_{i}\right)_{1, p} \\
\left(b_{i}, \beta_{i}\right)_{1, q}
\end{array}\right] f_{2}(t) d t
$$

both integrals converging, then

$$
f_{1}(t) \equiv f_{2}(t)
$$

\section{3}

In this section we shall establish a theorem concerning the transform given by (1.1) and the following transform introduced by Bhise [8, p. 1]

$$
\begin{aligned}
& G\left\{f(x) ; c_{1}, \cdots, c_{r} ; d_{1}, \cdots, d_{r}, \xi ; s\right\} \\
&\left.=s \int_{0}^{\infty} \begin{array}{r}
G_{m, m+1}^{m+1,0} \\
s x
\end{array} \mid \begin{array}{c}
c_{1}+d_{1}, \cdots, c_{r}+d_{r} \\
d_{1}, \cdots, d_{r}, \xi
\end{array}\right] f(x) d x .
\end{aligned}
$$


(3.1) itself is a very general transform and reduces to well known integral transforms as pointed out in the paper referred above.

THEOREM 1. If

$$
\phi(s)=H\left\{h(x) ;\left(a_{i}, \alpha_{i}\right)_{1, p} ;\left(b_{i}, \beta_{i}\right)_{1, q} ; s\right\},
$$

then

$$
s^{\rho+\sigma} \phi\left(s^{-\sigma}\right)=G\left\{t^{-\rho}\left[\int_{0}^{\infty} F(t, x) h(x) d x\right] ; c_{1}, \cdots, c_{r}, d_{1}, \cdots, d_{r} ; \xi ; s\right\}
$$

where

$$
F(t, x)=H_{p+r, q+r+1}^{m, n+r}\left[x t^{\sigma} \mid \begin{array}{l}
\left(\rho-d_{i}-c_{i}, \sigma\right)_{1, r},\left(a_{i}, \alpha_{i}\right)_{1, p} \\
\left(b_{i}, \beta_{i}\right)_{1, q},\left(\rho-d_{i}, \sigma\right)_{1, r},(\rho-\xi, \sigma)
\end{array}\right]
$$

provided that $h(x) \in A(\alpha, \beta, \gamma), R(\gamma)>0, R\left\{\sigma\left(b_{n} / \beta_{n}\right)-\rho+1\right\}>\max R\left\{-\xi,-d_{i}\right\}$ $(i=1, \cdots, r), \quad \sigma>0, \quad R(s)>0, \quad \lambda-\sigma>0, \quad\left(\lambda=\sum_{1}^{n} \alpha_{j}-\sum_{n+1}^{p} \alpha_{j}+\sum_{1}^{m} \beta_{j}-\right.$ $\left.\sum_{m+1}^{q} \beta_{j}\right), \quad R\left(\alpha+1+\left(b_{n} / \beta_{n}\right)\right)>0(h=1, \cdots, m), R(\beta+1)<\min R\left(\left(1-a_{j}\right) / \alpha_{j}\right)$, $\left.\left(1+d_{i}+c_{i}-\rho\right) / \sigma\right)(j=1, \cdots, n ; i=1, \cdots, r)$ and the G-function transform of

exists.

$$
t^{-\rho} \int_{0}^{\infty} F(t, x) h(x) d x
$$

PrOOF. Interpreting (3.2) with the help of (1.1), we have

$$
\phi(s)=s \int_{0}^{\infty} H_{p, q}^{m, n}\left[s x \mid \begin{array}{c}
\left(a_{i}, \alpha_{i}\right)_{1, p} \\
\left(b_{i}, \beta_{i}\right)_{1, q}
\end{array}\right] h(x) d x
$$

therefore

$$
s^{\rho+\sigma} \phi\left(s^{-\sigma}\right)=s^{\rho} \int_{0}^{\infty} H_{p, q}^{m, n}\left[x s^{-\sigma} \mid \begin{array}{l}
\left(a_{i}, \alpha_{i}\right)_{1, p} \\
\left(b_{i}, \beta_{i}\right)_{1, q}
\end{array}\right] h(x) d x
$$

Also from (1.7) it follows that

$$
\begin{aligned}
s^{\rho} H_{p, q}^{m, n}\left[x s^{-\sigma}\right. & \left.\begin{array}{l}
\left(a_{i}, \alpha_{i}\right)_{1, p} \\
\left(b_{i}, \beta_{i}\right)_{1, q}
\end{array}\right] \\
= & s \int_{0}^{\infty} t^{-\rho} H_{r, r+1}^{r+1,0}\left[\begin{array}{l}
s t \\
c_{1}+d_{1}, c_{2}+d_{2}, \cdots, c_{r}+d_{r} \\
d_{1}, \cdots, d_{r}, \xi
\end{array}\right] F(t, x) d x .
\end{aligned}
$$

Now on substituting the value of

$$
s^{\rho} H_{p, q}^{m, n}\left[x s^{-\sigma} \mid \begin{array}{l}
\left(a_{i}, \alpha_{i}\right)_{1, p} \\
\left(b_{i}, \beta_{i}\right)_{1, q}
\end{array}\right]
$$

from (3.6) in (3.5), inverting the order of integration therein (which is justified on account of the absolute convergence of the integrals involved under the conditions stated), we get the result by virtue of (3.1). 
Since the integral transforms involved in the above theorem are most general, it can yield deep and useful results. A number of theorems involving Laplace transform, Hankel transform, hypergeometric transform. etc and their various generalizations follow as special cases of the above theorem. For lack of space, however, we shall cite here only two cases.

(a) If we replace $m$ by $m+1, n$ by $0, p$ by $m, q$ by $m+1$ in the above theorem, put $\alpha$ 's and $\beta$ 's equal to unity, take $a_{1}=\eta_{1}+\delta_{1}, \cdots, a_{m}=\eta_{m}+\delta_{m} ; b_{1}=\eta_{1}, \cdots$, $b_{m}=\eta_{m}, b_{m+1}=\eta$ and $\sigma=N / S$ where $N$ and $S$ are $+v e$ integers, $F(t, x)$ given by (3.4) degenerates into Meijer's $G$-function [7, p. 40] and we get an important theorem recently given by Bhise $[8$, p. 2].

(b) Again if we put $m=1, n=p=0, q=2, b_{1}=v, \beta_{1}=1, b_{2}=-\lambda+\mu v$ and $\beta_{2}=\mu$ in (1.1), it reduces to the following theorem involving generalized Hankel transform introduced by Ram Kumar and referred to by the authors in their previous paper [3].

THEOREM 2. If

$$
\phi(s)=s \int_{0}^{\infty}(s x)^{v} J_{\lambda}^{\mu}(s x) h(x) d x,
$$

then

$s^{\rho+\sigma} \phi\left(s^{-\sigma}\right)=G\left\{t^{-\rho}\left(\int_{0}^{\infty} F(t, x) h(x) d x\right) ; c_{1}, \cdots, c_{r} ; d_{1}, \cdots, d_{r} ; \xi ; s\right\}$

where

$$
F(t, x)=H_{r, r+3}^{1, r}\left[x t^{\sigma} \mid \begin{array}{l}
\left(\rho-d_{i}-c_{i}, \sigma\right)_{1, r} \\
(v, 1),(-\lambda+\mu \nu, \mu),\left(\rho-d_{i}, \sigma\right)_{1, r},(\rho-\xi, \sigma)
\end{array}\right]
$$

under the conditions obtainable directly from the theorem 1.

On taking $d_{i}=0(i=1, \cdots, r), \xi=0$ in theorem 2, we get the following interesting relationship between Laplace transform and generalized Hankel transform under the conditions obtainable from theorem 1.

COROLLARY. If

$$
\phi(s)=s \int_{0}^{\infty}(s x)^{v} J_{\lambda}^{\mu}(s x) h(x) d x,
$$

then

$s^{\rho+\sigma} \phi\left(s^{-\sigma}\right)=L\left\{t^{-\rho}\left(\int_{0}^{\infty} h(x) H_{0,3}^{1,0}\left[x t^{\sigma} \mid(v, 1),(-\lambda+\mu v, \mu),(\rho, \sigma)\right] d x\right) ; s\right\}$

where

$$
L\{f(x) ; s\}=s \int_{0}^{\infty} e^{-s x} d x
$$




\section{References}

[1] A. Erdelyi et al., Higher transcendental functions Vol. I, (McGraw-Hill, New York, 1953).

[2] E. Lerch, 'Sur un point de la theorie des functions generatrices d'Abel', Acta Math. Stockh. 27 (1903), 339.

[3] K. C. Gupta and P. K. Mittal, 'The $H$-function transform', J. Australian Math. Soc. 11 (1970), 142-148.

[4] K. C. Gupta and S. S. Mittal, 'On Gauss's hypergeometric transform, Proc. Nat. Acad. Sc., India 37A (1967), 49-55.

[5] R. K. Saxena, Some theorems on generalized Laplace transform-I, Proc. Nat. Inst. Sci. India 26A (1960), 400-413.

[6] U. C. Jain, 'On an integral involving the $H$-function', J. Australian Math. Soc. 8 (1968), 373-376.

[7] U. C. Jain, A study of integral transforms (Thesis approved for Ph.D. degree, University of Udaipur, India, 1967).

[8] V. M. Bhise, 'Certain properties of Meijer-Laplace transform', Compositio Mathematica 18 (1967), 1-6.

Department of Mathematics

Malaviya Regional Engineering College

Jaipur-4, India

and

Department of Mathematics

Government College

Ajmer, India 